\title{
Histopathological Mapping of Meningiomas: A 10-year Retrospective Analysis
}

\author{
${ }^{1}$ Department of Pathology, Institute of Human Behaviour and \\ Allied Sciences, New Delhi, India \\ 2Department of Pathology, Institute of Human Behaviour and Allied \\ Sciences, New Delhi, India \\ ${ }^{3}$ Department of Neurosurgery, Guru Teg Bahadur Hospital, \\ University College of Medical Sciences, New Delhi, India
}

Ishita Pant ${ }^{1}$ Sujata Chaturvedi ${ }^{2}$ Pragyan Sarma $^{3}$ Gurbachan Singh ${ }^{3}$

\begin{abstract}
Address for correspondence Ishita Pant, MD, Department of Pathology, Institute of Human Behaviour and Allied Sciences, Dilshad Garden, New Delhi 110095, India (e-mail: ishitapant@gmail.com).
\end{abstract}

Indian J Neurosurg 2021;10:203-209.

\begin{abstract}
Background Meningiomas are a group of neoplasms showing a spectrum of histopathological variants, mostly corresponding to World Health Organization (WHO) grade I. However, certain histologic variants correspond histologically to WHO grades II and III. In this study, the histopathological spectrum of the meningiomas received in our department over the past 10 years is being revisited, with the aim to analyze the various histopathological variants, in order to assess their grade as per WHO 2016 criteria and also assess their epidemiology and localization.

Materials and Methods A retrospective analysis of the histopathology records and clinical case files was done in 302 cases of histopathologically diagnosed cases of intracranial/intraspinal meningiomas (WHO grade I, II and III) received over a period of 10 years in our department. Hematoxylin and eosin (HE) staining was done for histological typing and grading of the tumors, and the cases were analyzed as per WHO 2016 criterion. Age and sex distribution along with the localization of the 302 cases was also analyzed.

Results Among these, grade I meningiomas (93.05\%) were the most common. According to histopathological type, transitional meningioma (76.50\%) was the most common. Maximum cases were located at the cerebral convexities, reported in the fifth decade with a female preponderance.

Keywords

- Meningioma

- WHO grade

- histopathological variant

Conclusion As per WHO 2016, grade I meningiomas are associated with low-risk of recurrence and aggressive behavior, while WHO grade II and III meningiomas are associated with greater likelihood of recurrence and aggressive behavior. This classification itself summarizes the importance of accurate typing and grading of meningiomas on histopathology.
\end{abstract}

\section{Introduction}

Meningiomas are a group of neoplasms arising from meningothelial (arachnoid) cells in the leptomeninges. These cells have both epithelial and mesenchymal characteristics, reflected by the spectrum of meningiomas exhibiting diverse histologic appearances. Histopathological variants of

published online May 13, 2021
DOI https://doi.org/ 10.1055/s-0040-1718990 ISSN 2277-954X. meningiomas mostly correspond to WHO grade I. However, certain histopathologic variants correspond to WHO grades II and III. WHO 2016 has classified these into meningiomas with low-risk of recurrence and aggressive behavior (WHO grade I) and meningiomas with greater likelihood of recurrence and aggressive behavior (WHO grade II and grade III). ${ }^{1}$

(c) 2021. Neurological Surgeons' Society of India.

This is an open access article published by Thieme under the terms of the Creative Commons Attribution-NonDerivative-NonCommercial-License, permitting copying and reproduction so long as the original work is given appropriate credit. Contents may not be used for commercial purposes, or adapted, remixed, transformed or built upon. (https://creativecommons.org/licenses/by-nc-nd/4.0/) Thieme Medical and Scientific Publishers Pvt. Ltd., A-12, 2nd Floor, Sector 2, Noida-201301 UP, India 
Meningiomas constitute approximately 28 to $30 \%$ of primary central nervous system (CNS) tumors and account for $15 \%$ of intracranial tumors and approximately $25 \%$ of intraspinal tumors. ${ }^{2}$ They occur most commonly in middle-aged patients, with a peak during the sixth and seventh decades. ${ }^{3}$ They show an obvious female predominance, with the male:female ratio being approximately $1: 1.7$; at the same time, it has been reported that grade II and III meningiomas occur at a higher rate in males. ${ }^{4}$ The vast majority of meningiomas arise in intracranial, intraspinal or orbital locations. Intraventricular and epidural locations are uncommon.

In this study, the histopathological spectrum of the meningiomas received in our department over the past 10 years is being revisited with the aim to analyze the various histopathological variants, in order to assess their grade as per WHO 2016 criteria and also assess their epidemiology and localization.

\section{Materials and Methods}

A retrospective analysis of the histopathology records and clinical case files was done in 302 cases of histopathologically diagnosed cases of intracranial and intraspinal meningiomas (WHO grade I, II and III) received over a period of 10 years (from 2009-2018) in our department. These cases were received from the neurosurgery departments of two superspecialty government hospitals, Institute of Human Behavior and Allied Sciences and Guru Teg Bahadur Hospital in east Delhi, India.

In all, there were 175/302 cases where both intraoperative fresh unfixed and later formalin-fixed tissue samples were received. Intraoperative reporting was done on the basis of crush smears and frozen sections. For crush smears, toluidine blue staining was done as the first choice, and if additional smears were available, hematoxylin and eosin (HE) staining was also applied. Agreement between intraoperative and final histopathologic diagnosis was recorded. The specimens received in formalin for paraffin sections for all 302 cases were stained by HE and were further analyzed for histopathological typing and grading as per WHO 2016 criterion. Immunohistochemical stains were applied only in those cases where it was required to supplement the histopathological diagnosis established by HE stain.

In addition, age and gender distribution along with the localization of the 302 cases was also analyzed.

\section{Results}

Amongst the 302 meningioma cases, based on WHO 2016 grading criteria, grade I meningiomas (281 cases) were the most common, representing $93.05 \%$ of cases, followed by 16 cases of grade II $(5.30 \%)$ and five cases $(1.65 \%)$ of grade III meningiomas ( - Table $\mathbf{1}$ ). According to histological type, amongst the grade I, 231 meningiomas were transitional (76.50\%), 11 meningothelial (3.64\%), 11 angiomatous (3.64\%), 8 psammomatous (2.65\%), 8 fibroblastic $(2.65 \%), 7$ microcystic (2.32\%), 3 metaplastic (0.99\%), and 2 secretory $(0.66 \%)$ ( - Fig. 1A). Among grade II, there were 15 cases of atypical
Table 1 Table showing histopathological variants and grades of meningiomas

\begin{tabular}{|c|c|c|c|}
\hline Histopathological variants & $\begin{array}{l}\text { WHO } \\
\text { grade }\end{array}$ & $\begin{array}{l}\text { Number } \\
\text { of cases }\end{array}$ & $\%$ of cases \\
\hline Transitional meningioma & 1 & 231 & 76.50 \\
\hline Meningothelial meningioma & I & 11 & 3.64 \\
\hline Angiomatous meningioma & I & 11 & 3.64 \\
\hline Fibrous (fibroblastic) meningioma & I & 8 & 2.65 \\
\hline Psammomatous meningioma & 1 & 8 & 2.65 \\
\hline Microcystic meningioma & I & 7 & 2.32 \\
\hline Metaplastic meningioma & 1 & 3 & 0.99 \\
\hline Secretory meningioma & I & 2 & 0.66 \\
\hline \multicolumn{2}{|l|}{ WHO grade-I meningiomas } & 281 & 93.05 \\
\hline Atypical meningioma & II & 15 & 4.97 \\
\hline Clear cell meningioma & II & 1 & 0.33 \\
\hline \multicolumn{2}{|l|}{ WHO grade-II meningiomas } & 16 & 5.30 \\
\hline Rhabdoid meningioma & III & 3 & 0.99 \\
\hline Anaplastic meningioma & III & 2 & 0.66 \\
\hline \multicolumn{2}{|l|}{ WHO grade-III meningiomas } & 5 & 1.65 \\
\hline
\end{tabular}

Abbreviation: WHO, World Health Organization.

meningiomas (4.97\%), and 1 case of clear cell variant $(0.33 \%)$ ( - Fig. 1B). Among grade III, 3 cases were of rhabdoid meningioma $(0.99 \%)$ and 2 were anaplastic meningioma $(0.66 \%)$ (-Figs. 1C and 2).

Overall, the mean age was 44.58 years (range: 9 to 78 years). A maximum of 81 cases $(26.82 \%)$ were reported in the fifth decade, followed by 68 cases $(22.52 \%)$ in the fourth decade, 61 cases $(20.20 \%)$ in the sixth decade, 43 cases $(14.24 \%)$ in the third decade, 30 cases (9.93\%) in the seventh decade, 15 cases $(4.97 \%)$ in the second decade, and 3 cases $(0.99 \%)$ in the eighth decade. A total of 6 cases $(1.99 \%)$ were reported in the pediatric age group, ranging from 9 to 16 years. There were 81 males (26.82\%) and 221 female patients (73.18\%). In all cases, including aggressive variants, there was a female predominance, with the male:female ratio being $1: 2.5$. Among the 302 cases, 252 cases $(84.11 \%)$ were found at the intracranial location, followed by 48 cases (15.89\%) found at the intraspinal location. Among the intracranial meningiomas, 155 cases (51.32\%) were at the cerebral convexities, followed by 45 cases at posterior fossa (14.90\%), 22 cases (7.28\%) at sphenoid ridges, 10 cases (3.31\%) at parasellar/ suprasellar regions, 7 cases $(2.32 \%)$ at olfactory grooves, 5 cases $(1.66 \%)$ at tentorium, and 2 cases $(0.66 \%)$ at the optic nerve sheath. Regarding the intraspinal location, a maximum of 36 cases $(75.0 \%)$ were found in the thoracic region, followed by 12 cases $(25.0 \%)$ in the cervical region ( - Fig. 3 ; - Table 2 ).

Among the grade I meningiomas, transitional meningiomas belonged to the age range of 9 to 78 years, with a mean age of 44.83 years. The male:female ratio was 1:3.5 and the most common location was cerebral convexities. Meningothelial meningiomas were in the age range of 32 to 75 years with a mean age of 50.81 years. The male:female ratio was $1: 1.2$ and the most common location was cerebral convexities. Angiomatous meningiomas were in the age range of 

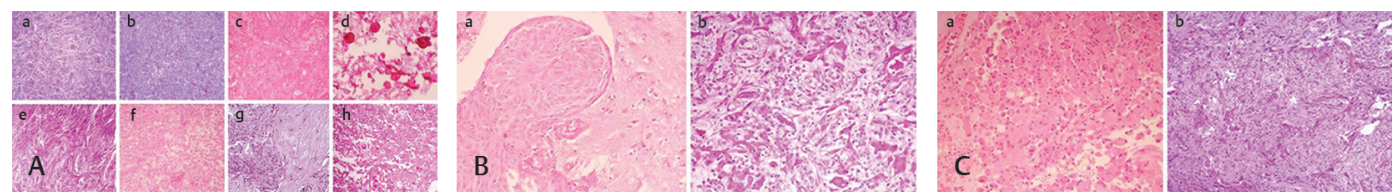

Fig. 1 (A) Histopathology showing (a) transitional meningioma (HE; $\times 200)$; (b) meningothelial meningioma (HE; $\times 200)$; (c) angiomatous meningioma (HE; $\times 200)$; (d) psammomatous meningioma (HE; $\times 200)$; (e) fibroblastic meningioma (HE; $\times 200) ;($ f) microcystic meningioma (HE; $\times 200)$; (g) metaplastic meningioma (HE; $\times 200)$; (h) secretory meningioma (HE; $\times 200)$. (B) Histopathology showing (a) atypical meningioma (HE; $\times 200)$; (b) clear cell meningioma (HE; $\times 200)$. (C) Histopathology showing (a) rhabdoid meningioma (HE; $\times 200) ;($ b) anaplastic meningioma $(\mathrm{HE} ; \times 200)$. HE, hematoxylin and eosin.

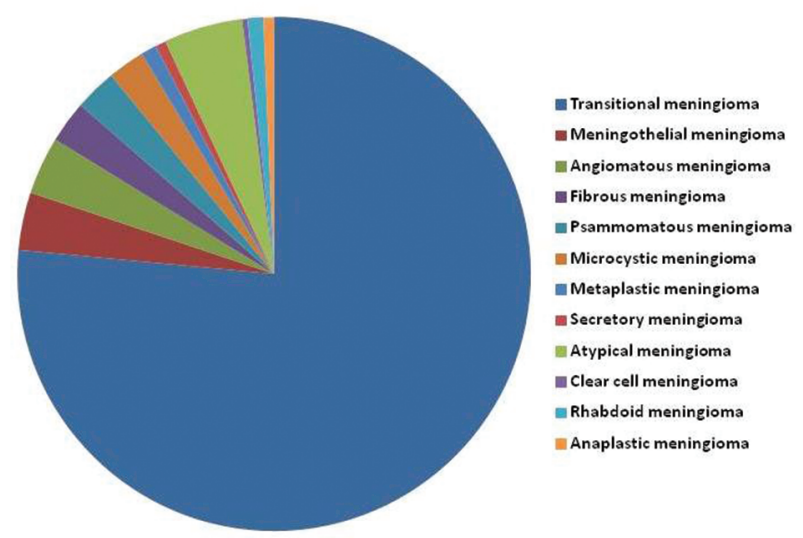

Fig. 2 Pie chart showing the frequency of meningioma variants.

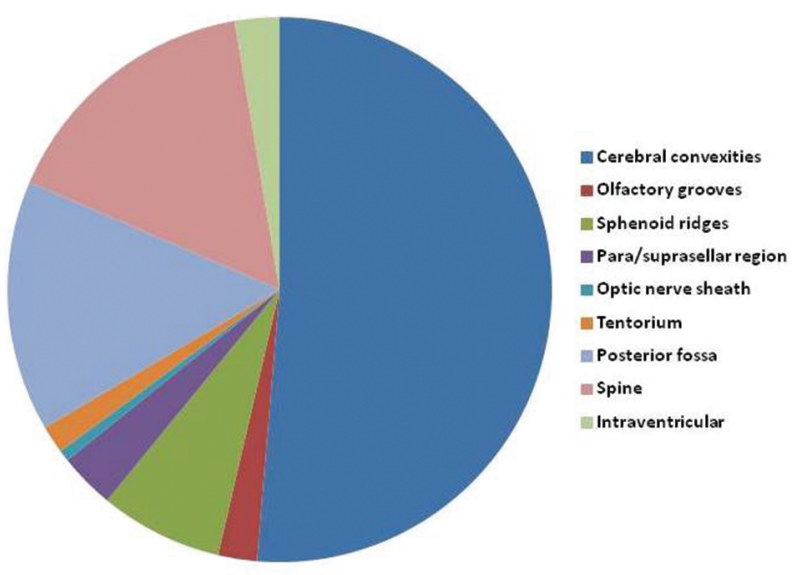

Fig. 3 Pie chart showing the distribution of meningioma as per various locations.

25-60 years with a mean age of 41.81 years. The male:female ratio was $1: 1.7$ and the most common location was cerebral convexities. Fibrous meningiomas were in the age range of 22 to 50 years with a mean age of 35.75 years. The male: female ratio was $1: 3$ and the most common location was cerebral convexities. Psammomatous meningiomas were in the age range of 20 to 60 years with a mean age of 44.75 years. The male:female ratio was 1:3.5 and the most common locations included cerebral convexities and thoracic spine. Microcystic meningiomas were in the age range of 28 to 64 years with a mean age of 47.14 years. The male:female ratio was 1.3:1 and the most common location was cerebral convexities. Metaplastic meningiomas were in the age range of 44 to 51 years with a mean age of 48.67 years. The male:female ratio
Table 2 Table showing location-wise distribution

\begin{tabular}{|c|c|c|}
\hline Location & $\begin{array}{l}\text { Number } \\
\text { of cases }\end{array}$ & $\begin{array}{l}\% \text { of } \\
\text { cases }\end{array}$ \\
\hline \multicolumn{3}{|l|}{ Common location } \\
\hline $\begin{array}{l}\text { Cerebral convexities (parasagit- } \\
\text { tal, falx, and venous sinuses }\end{array}$ & 155 & 51.32 \\
\hline Olfactory grooves & 7 & 2.32 \\
\hline Sphenoid ridges & 22 & 7.28 \\
\hline Para/suprasellar regions & 10 & 3.31 \\
\hline Optic nerve sheath & 2 & 0.66 \\
\hline Tentorium & 5 & 1.66 \\
\hline Posterior fossa & 45 & 14.90 \\
\hline Spine (cervical and thoracic) & 48 & 15.90 \\
\hline \multicolumn{3}{|l|}{ Uncommon location } \\
\hline $\begin{array}{l}\text { Intraventricular (lateral ventricle } \\
\text { and third ventricle) }\end{array}$ & 8 & 2.65 \\
\hline
\end{tabular}

was 2:1 and the most common location was cerebral convexities. Secretory meningiomas manifested at the age of 50 years with a male:female ratio of $0: 1$. The most common locations included olfactory grooves and sphenoid ridges ( - Table 3 ).

Among the grade II meningiomas, atypical meningiomas were in the age range of 18 to 60 years with a mean age of 41.86 years. The male:female ratio was $1.1: 1$ and the most common location was cerebral convexities. Clear cell meningiomas had a mean age of 30 years. The male:female ratio was 1:0 and the most common location was thoracic spine. Among grade III meningiomas, rhabdoid meningiomas were in the age range of 13 to 18 years with a mean age of 16.33 years. The male:female ratio was $0: 1$ and the most common location was cerebral convexities. Anaplastic meningiomas were in the age range of 22 to 38 years with a mean age of 30 years. The male:female ratio was 1:0 and the most common location was cerebral convexities (-Table $\mathbf{3}$ ).

Among the 175 cases where agreement between intraoperative reporting and final histopathologic diagnosis was done, 160 cases showed complete agreement, while 15 cases showed disagreement between the intraoperative reporting and the final histopathologic diagnosis. The retrospective analysis revealed that maximal disagreement was recorded among the high-grade meningiomas, mostly labeled as high-grade tumors during the intraoperative reporting with no comment on cell lineage.

In addition, the authors also performed a detailed anatomical mapping of various histopathological variants along with gender distribution ( - Table 4$)$. 
Table 3 Clinicopathological details of various variants of meningioma

\begin{tabular}{|l|l|l|l|l|l|}
\hline Histopathological variant ( $n)$ & Age range (y) & Mean age (y) & $\begin{array}{l}\text { M:F } \\
\text { ratio }\end{array}$ & Most common location \\
\hline \multicolumn{5}{|c|}{ Meningiomas (WHO grade I) } & with low risk of recurrence and aggressive behavior \\
\hline Transitional meningioma (231) & $9-78$ & 44.83 & $1: 3.5$ & Cerebral convexities \\
\hline Meningothelial meningioma (11) & $32-75$ & 50.81 & $1: 1.2$ & Cerebral convexities \\
\hline Angiomatous meningioma (11) & $25-60$ & 41.8 & $1: 1.7$ & Cerebral convexities \\
\hline Fibrous (fibroblastic) meningioma (8) & $22-50$ & 35.75 & $1: 3$ & Cerebral convexities \\
\hline Psammomatous meningioma (8) & $20-60$ & 44.75 & $1: 3$ & Cerebral convexities, spine \\
\hline Microcystic meningioma (7) & $28-64$ & 47.14 & $1.3: 1$ & Cerebral convexities \\
\hline Metaplastic meningioma (3) & $44-51$ & 48.67 & $2: 1$ & Cerebral convexities \\
\hline Secretory meningioma (2) & 50 & 50 & $0: 1$ & Olfactory grooves, sphenoid ridges \\
\hline \multicolumn{2}{|c|}{ Meningiomas (WHO grade II and grade III) with high risk of recurrence and aggressive behavior } \\
\hline Atypical meningioma (15) & $18-60$ & 41.86 & $1.1: 1$ & Cerebral convexities \\
\hline Clear cell meningioma (1) & 30 & 30 & $1: 0$ & Spine \\
\hline Rhabdoid meningioma (3) & $13-18$ & 16.33 & $0: 1$ & Cerebral convexities \\
\hline Anaplastic meningioma (2) & $22-38$ & 30 & $1: 0$ & Cerebral convexities \\
\hline
\end{tabular}

Abbreviation: WHO, World Health Organization.

Table 4 Anatomical mapping of various histopathological variants of meningioma along with the gender distribution

\begin{tabular}{|c|c|c|c|c|c|c|c|c|c|c|c|c|c|c|c|c|c|c|c|c|c|c|c|c|}
\hline \multirow[t]{2}{*}{ Location } & \multicolumn{24}{|c|}{ Histopathological variants } \\
\hline & \multicolumn{2}{|c|}{ TM } & \multicolumn{2}{|c|}{ MM } & \multicolumn{2}{|c|}{ AM } & \multicolumn{2}{|c|}{ FM } & \multicolumn{2}{|c|}{ PM } & \multicolumn{2}{|c|}{ Mi M } & \multicolumn{2}{|c|}{ Me M } & \multicolumn{2}{|c|}{ SM } & \multicolumn{2}{|c|}{ At $M$} & \multicolumn{2}{|c|}{ CCM } & \multicolumn{2}{|c|}{ RM } & \multicolumn{2}{|c|}{ Ana M } \\
\hline \multirow{2}{*}{$\begin{array}{l}\text { Cerebral con- } \\
\text { vexities (paras- } \\
\text { agittal, falx, } \\
\text { and venous } \\
\text { sinuses }\end{array}$} & \multicolumn{2}{|c|}{112} & \multicolumn{2}{|c|}{11} & & & \multicolumn{2}{|c|}{8} & \multicolumn{2}{|c|}{8} & \multicolumn{2}{|c|}{7} & \multicolumn{2}{|c|}{3} & \multicolumn{2}{|c|}{2} & & & \multicolumn{2}{|c|}{1} & \multicolumn{2}{|c|}{3} & & \\
\hline & 31 & 81 & 5 & 6 & & & 2 & 6 & 2 & 6 & 4 & 3 & 1 & 2 & 0 & 2 & & & 1 & 0 & 0 & 3 & & \\
\hline \multirow{2}{*}{$\begin{array}{l}\text { Olfactory } \\
\text { grooves }\end{array}$} & \multicolumn{2}{|c|}{7} & & & & & & & & & & & & & & & & & & & & & & \\
\hline & 3 & 4 & & & & & & & & & & & & & & & & & & & & & & \\
\hline \multirow{2}{*}{$\begin{array}{l}\text { Sphenoid } \\
\text { ridges }\end{array}$} & & & & & & & & & & & & & & & & & & & & & & & & \\
\hline & 5 & 18 & & & & & & & & & & & & & & & & & & & & & & \\
\hline Para/suprasellar & & & & & & & & & & & & & & & & & & & & & & & & \\
\hline regions & 2 & 8 & & & & & & & & & & & & & & & & & & & & & & \\
\hline Optic nerve & & & & & & & & & & & & & & & & & & & & & & & & \\
\hline & 0 & 2 & & & & & & & & & & & & & & & & & & & & & & \\
\hline Tentorium & & & & & & & & & & & & & & & & & & & & & & & & \\
\hline & 1 & 3 & & & & & & & & & & & & & & & & & & & & & 1 & 0 \\
\hline Posterior fossa & & & & & 1 & 1 & & & & & & & & & & & 1 & & & & & & & \\
\hline & & 18 & & & & 7 & & & & & & & & & & & & 7 & & & & & & \\
\hline Spine (cervical & & & & & & & & & & & & & & & & & & & & & & & & \\
\hline and thoracic) & 8 & 40 & & & & & & & & & & & & & & & & & & & & & & \\
\hline Intraventricular & & & & & & & & & & & & & & & & & & & & & & & 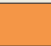 & \\
\hline $\begin{array}{l}\text { (lateral/third } \\
\text { ventricle) }\end{array}$ & 1 & 5 & & & & & & & & & & & & & & & & & & & & & 1 & 0 \\
\hline
\end{tabular}

Abbreviations: AM, angiomatous meningioma; Ana M, anaplastic meningioma; Aty M, atypical meningioma; CCM, clear cell meningioma; FM, fibrous meningioma; Me M, metaplastic meningioma; Mi M, microcystic meningioma; MM, meningothelial meningioma; PM, psammomatous meningioma; RM, rhabdoid meningioma; SM, secretory meningioma; TM, transitional meningioma.

$\begin{aligned} & \text { Number } \\ & \text { Male } \\ & \text { Female } \\ & \text { Nill }\end{aligned}$




\section{Discussion}

Harvey Cushing proposed the term meningioma in 1922, based primarily on anatomy. Over the years, various hypotheses on the cell of origin were proposed, including dural, endothelial, fibroblastic and epithelial cell types. After a long debate, a possibility was raised that these tumors might originate from the inner arachnoidal lining of the dura, rather than the dense fibrous tissue of dura. It was also identified that the histopathology of meningiomas was similar to the cell clusters that line arachnoid villi, and since then, these arachnoidal cap cells have been considered the cell of origin for meningiomas. ${ }^{5}$

In 1938, Harvey Cushing with Louise Eisenhardt published a detailed monograph on 313 meningiomas, providing a better understanding of these tumors. In continuum, over the next few decades, several variants were identified with the application of newer techniques like karyotyping, electron microscopy and immunohistochemistry. In 1982, a second major monograph summarizing the major advances based on 1300 cases of meningiomas was published by John Kepes. ${ }^{6}$ Since then, over the years, a few more aggressive variants were identified and added to the existing variants; the grading system was extensively revised and, gradually with the advent of molecular pathology, the World Health Organization's (WHO) 2007 classification was introduced. ${ }^{7}$ In 2016, WHO updated the classification and grading of tumors of the CNS, incorporating well-established molecular parameters and classified meningiomas into meningiomas with low-risk of recurrence and aggressive behavior (WHO grade I) and meningiomas with greater likelihood of recurrence and aggressive behavior (WHO grade II and grade III). ${ }^{1}$

Meningiomas account for approximately $20 \%$ to $30 \%$ of primary intracranial neoplasms. These are most common in middle aged to elderly adults, showing a peak in the sixth and seventh decades. ${ }^{3}$ In addition, meningiomas are also seen in the pediatric population, ${ }^{8}$ although meningiomas of the first two decades are extremely uncommon. ${ }^{9}$ In our study, the peak was seen in the fifth decade, followed by the fourth decade, concordant to the studies conducted by Shah et al, Shrilakshmi, and Patty. ${ }^{10-12}$ In our study, we found 6 cases $(1.99 \%)$ in the pediatric age group, results almost similar to the study conducted by Mehta et al, in which they had reported $1.92 \%$ pediatric meningiomas. ${ }^{13}$ These tumors are more common in women than men, with an approximate 1.7:1 female:male ratio. In our study, the female:male ratio was 2.5:1, similar to the results of Thomas et al. ${ }^{14}$ This difference is greatest prior to menopause, with the highest female:male ratio of 3.15:1. Grade II and III meningiomas are reported at higher rates in males. ${ }^{4}$

The vast majority of meningiomas arise in intracranial, intraspinal or orbital locations. Within the cranial cavity, common sites include the cerebral convexities, olfactory grooves, sphenoid ridges, para/suprasellar regions, optic nerve sheath, petrous ridges, tentorium and posterior fossa. ${ }^{8}$ In this study, we found $81.46 \%$ intracranial and19.3\% intraspinal meningiomas. Among the intracranial meningiomas, the maximum numbers were located over the cerebral convexities, followed by the sphenoid ridges, while among the intraspinal meningiomas, the most common location was the thoracic spine, concordant with the existing literature and the study conducted by Shrilakshmi. ${ }^{11}$ Intraventricular and epidural locations are uncommon. This study showed eight intraventricular cases. Rare examples have been reported outside the neural axis, ${ }^{15}$ although our study did not show any case outside the neural axis.

Clinical manifestations depend on the location of the tumor. Since these are slow growing tumors, usually the symptoms occur due to the compression of the adjacent structures like focal neurological deficits, increased intracranial pressure, headache and seizures.

MRI shows typical circumscribed, isodense, dural masses showing uniform contrast enhancement. Meningiomas may show calcification and evidence of bone or cartilage. Calcification is best seen on CT scan. The characteristic radiologic feature of meningioma is the dural tail, a wedge-shaped extension of tumor at the edge which is contrast enhancing. ${ }^{16}$ Higher grades do occasionally show peritumoral cerebral edema. ${ }^{17}$

Macroscopically, meningiomas show a diverse range, depending upon the location, growth pattern and the histological variant. Mostly, these are rubbery, well-circumscribed, spherical and firmly attached to the inner surface of the dura. Commonly, these are solitary neoplasm, but sporadic cases may show multiple meningiomas. Occasionally, these are dumbbell-shaped, and rarely these may be en plaque meningiomas, defined by a carpet like growth pattern, resulting in long stretches of thickened, sometimes rough or shaggy dura. ${ }^{18}$ The tumor surface is usually smooth or bosselated like cauliflower. The cut surface is white, yellow or tan, often showing white streaky bands of fibrous tissue. Cut surface is variable in different variants, like it is usually gritty in psammomatous variant, may be yellow in xanthomatous variant, may be white in metaplastic variant due to cartilage and bone formation, a moist glistening surface usually indicates microcystic variant, whereas the secretory variant has a tan red gland-like appearance. Foci of hemorrhage and necrosis on cut surface suggest variants like atypical and anaplastic.

Meningioma variants in 2016 have been grouped by WHO grade and biological behavior into (i) meningiomas with low-risk of recurrence and aggressive behavior and (ii) meningiomas with greater likelihood of recurrence and aggressive behavior. The first group comprises meningothelial meningioma, fibroblastic meningioma, transitional meningioma, psammomatous meningioma, angiomatous meningioma, microcystic meningioma, secretory meningioma, lymphocyte-rich meningioma and metaplastic meningioma, while the second group comprises chordoid meningioma, clear cell meningioma, atypical meningioma, papillary meningioma, rhabdoid meningioma, anaplastic meningioma and meningiomas of any subtype with high-proliferation index. In the first group, all variants correspond to WHO grade I; however, in the second group, chordoid meningioma, clear cell meningioma, atypical meningioma correspond to WHO grade II and papillary meningioma, rhabdoid meningioma, anaplastic meningioma correspond to WHO 
grade III. ${ }^{1}$. In our study, the most common histopathological variant was transitional meningioma (76.50\%), similar to the results published by Babu et al, Grondahl et al, Veeramani et al and Shanthi et al. ${ }^{14,19-21}$ All these authors had reported transitional meningioma as the most common variant in their studies. In our study, 93.05\% cases were grade I, totally in concordance to the existing literature.

Meningiomas are basically surgically treated neoplasms. As far as the management is concerned, the best therapeutic approach is surgery, although radiation is also a major treatment modality, especially for patients for whom surgery carries high risk. ${ }^{22}$ Adjuvant radiotherapy has been shown to reduce recurrence rates in subtotally resected or higher grade meningiomas. Additional therapeutic modalities like stereotactic radiosurgery ("gamma knife"), fractionated stereotactic, intensity-modulated and proton beam forms of radiotherapy have been in use for smaller tumors or postsurgical residual masses.

Prognosis depends upon certain clinical factors, histopathology and grading and progesterone receptor (PR) status. The major clinical factors in recurrence include extent of resection, extent of invasion, and attachment to vital intracranial structures..$^{23,24}$ In terms of histopathology and grading, some histological variants are more likely to recur. However, overall WHO grade is the most useful morphological predictor of recurrence. Benign (WHO grade I) meningiomas have recurrence rates of approximately 7 to $25 \%$, whereas atypical meningiomas (WHO grade II) recur in 29 to $52 \%$ of cases and anaplastic meningiomas (WHO grade III) recur in 50 to $94 \%$ of cases. ${ }^{25}$ PR expression is quite variable, but usually it is inversely associated with meningioma grade. Generally, its absence negatively impacts disease free intervals, but at the same time, there remains great overlap in PR status among the WHO grades. ${ }^{26}$ Thus, PR expression is not independent of other known prognostic factors such as grade, and the significance of this finding in the absence of other prognostic features should not be overstated.

\section{Conclusion}

Meningiomas have a diverse spectrum of several histopathological variants corresponding to WHO grades I to III. WHO 2016 categorization of meningiomas into grade I (meningiomas associated with low-risk of recurrence and aggressive behavior) and grades II and III (meningiomas associated with greater likelihood of recurrence and aggressive behavior) itself summarizes the importance of accurate typing and grading of meningiomas on histopathology.

\section{Authors' Contributions}

I.P. conducted concepts design, definition of intellectual content, literature search, data analysis, manuscript preparation, and manuscript editing. S.C. helped in concepts design, definition of intellectual content, data analysis, manuscript preparation, and manuscript editing. P.S. facilitated data acquisition, manuscript preparation, and manuscript editing. G.S. helped in data acquisition and manuscript editing.

\section{Conflict of Interest}

None declared.

\section{References}

1 Perry A, Louis DN, Budka H, et al. Meningioma. In: Louis DN, Ohgaki H, Wiestler OD, Cavenee WK,eds. WHO Classification of Tumours of the Central Nervous System, Lyon; IARC 2016: 232-245

2 Bondy M, Ligon BL. Epidemiology and etiology of intracranial meningiomas: a review. J Neurooncol 1996;29(3):197-205

3 Dolecek TA, Propp JM, Stroup NE, Kruchko C. CBTRUS statistical report: primary brain and central nervous system tumors diagnosed in the United States in 2005-2009. Neuro-oncol 2012;14(Suppl 5) :v1-v49

4 Wiemels J, Wrensch M, Claus EB. Epidemiology and etiology of meningioma. J Neurooncol 2010;99(3):307-314

5 Cushing H, Eisenshardt L, Meningiomas: Their classification, Regional Behaviour, Life history and Surgical end results. Springfield, IL: CC Thomas; 1938

6 Kepes J, Meningiomas: Biology, Pathology and Differential Diagnosis. New York: Masson; 1982: 44-47

7 Perry A, Louis DN, Scheithauer BW, Meningiomas. In Louis DN, Ohgaki H, Wiestler OD, Cavenee WK, eds. WHO Classification of Tumours of the Central Nervous System, Lyon; IARC 2007: 164-172

8 Louis DN, Ohgaki H, Wiestler OD, et al. The 2007 WHO classification of tumours of the central nervous system. Acta Neuropathol 2007;114(2):97-109

9 Germano IM, Edwards MS, Davis RL, Schiffer D. Intracranial meningiomas of the first two decades of life. J Neurosurg 1994;80(3):447-453

10 Shah SR, Gonsai N, Makwana R. Histopathological study of meningioma in civil hospital, Ahmedabad. International Journal of Current Research and Review. 2013;5:76-82

11 Shrilakshmi S. Meningiomas:a clinicopathological study. Int J Med Res Health Sci. 2015;4:827-831

12 Patty IS. Central nervous system tumours- A clinicopathological study. J Dohuk Univ 2008;11:173-180

13 Mehta N, Bhagwati S, Parulekar G. Meningiomas in children: a study of 18 cases. J Pediatr Neurosci 2009;4(2):61-65

14 Backer-Grøndahl T, Moen BH, Torp SH. The histopathological spectrum of human meningiomas. Int J Clin Exp Pathol 2012;5(3):231-242

15 Mubeen B, Makhdoomi R, Nayil K, et al. Clinicopathological characteristics of meningiomas: experience from a tertiary care hospital in the Kashmir Valley. Asian J Neurosurg 2019; $14(1): 41-46$

16 Engelhard HH. Progress in the diagnosis and treatment of patients with meningiomas. Part I: diagnostic imaging, preoperative embolization. Surg Neurol 2001;55(2):89-101

17 Zhang H, Rödiger LA, Shen T, Miao J, Oudkerk M. Perfusion MR imaging for differentiation of benign and malignant meningiomas. Neuroradiology 2008;50(6):525-530

18 Khursheed N, Rumana M, Ramzan A, Abrar W. En-plaque spinal meningioma: A rare entity. Neurosurg 2013;23:61-63

19 Babu S, Uppin SG, Uppin MS, et al. Meningiomas: correlation of Ki67 with histological grade. Neurol India 2011;59(2):204-207

20 Veeramani T, Maheshwari J. Histopathological analysis and correlation of Ki 67 and progesterone receptor status with WHO grading in meningiomas. Annals of Pathology and Laboratory Medicine 2017;4:714-720

21 Shanthi V, Grandhi B, Rao NM, Rao BSS, Reddy VLM. Assessing the prognostic importance of ER, PR expression in meningiomas by comparing with proliferative rate using Ki67. Indian Journal of Pathology: Reserach and Practice 2017;6: 431-434 
22 McMullen KP, Stieber VW. Meningioma: current treatment options and future directions. Curr Treat Options Oncol 2004;5(6):499-509

23 Jääskeläinen J. Seemingly complete removal of histologically benign intracranial meningioma: late recurrence rate and factors predicting recurrence in 657 patients. A multivariate analysis. Surg Neurol 1986;26(5):461-469

24 Aizer AA, Bi WL, Kandola MS, et al. Extent of resection and overall survival for patients with atypical and malignant meningioma. Cancer 2015;121(24):4376-4381
25 Marciscano AE, Stemmer-Rachamimov AO, Niemierko A, et al. Benign meningiomas (WHO Grade I) with atypical histological features: correlation of histopathological features with clinical outcomes. J Neurosurg 2016;124(1):106-114

26 Roser F, Nakamura M, Bellinzona M, Rosahl SK, Ostertag H, Samii M. The prognostic value of progesterone receptor status in meningiomas. J Clin Pathol 2004;57(10):1033-1037 